\title{
Light Propagation on a Moving Closed Contour and the Role of Simultaneity in Special Relativity
}

\author{
Gianfranco Spavieri
}

\section{ABSTRACT}

We consider an example of a moving closed contour and the role played by simultaneity in the description of light propagation on the contour's moving sections. We show that, when constrained to propagate along the contour, the local speed of light on a moving section is no longer arbitrary and a consistent description requires conservation of simultaneity.

Keywords: Einstein synchronization, light propagation, one-way speed of light, relative simultaneity, Sagnac effect.

\author{
Published Online: August 30, 2021 \\ ISSN: $2684-4451$ \\ DOI : 10.24018/ejphysics.2021.3.4.99
}

\section{Gianfranco Spavieri*}

Centro de Física Fundamental, Universidad de Los Andes, Mérida, 5101 Venezuela.

(e-mail: gspavieri@gmail.com)

*Corresponding Author

\section{INTRODUCTION}

According to several authors [1]-[10] difficulties emerge with the use of the Lorentz transformations (LT) - based on standard Einstein synchronization and nonconservation of simultaneity - when applied to light propagation on a moving closed contour. The mentioned difficulties have been considered in some of the diverse interpretations of the Sagnac effect [11], [2]-[10], widely discussed in the literature [12]. In order to overcome the difficulties, some of these authors [1]-[6], [9]-[11] clarify the convenience of adopting transformations based on conservation of simultaneity (absolute simultaneity), in lieu of the LT based on relative simultaneity.

Although there are no difficulties with light propagation along open contours, in the case of a closed contour the root of the problem is the time discontinuity in the LT. As Landau and Lifshitz [10] put it: "... synchronization of clocks along a closed contour turns out to be impossible in general. In fact, starting out along the contour and returning to the initial point, we would obtain for $d x^{\circ}$ a value different from zero...". In line with the remark of Landau and Lifshitz [10] there is general agreement [1]-[6], [9]-[11] that the standard procedure proposed by Einstein fails (or is nonintegrable) when applied to the closed contour of the Sagnac effect.

We highlight the problem emerging with the LT by considering light propagation on the moving closed contour described in Sections II, III, and IV, where a section of the moving contour is in motion relative to the measuring device (e.g., a clock). In our novel example, light propagation can be described using Cartesian coordinates, where the different roles played by absolute and relative simultaneity are exposed. The length of the closed contour and the round-trip time $\tau_{\text {round }}$, taken by light to cover it and measured by the single clock, are independent of the choice of clock synchronization. Then, we find that, in the interval $\tau_{\text {round }}$ and at local speed $c$ on every relatively moving section, light can cover an open section only of the contour. If we require the contour covered by light to be closed, the speed of light is no longer conventional, and the determined synchronization corresponds to absolute synchrony and simultaneity. In Section V the results of our example are linked to the descriptions of light propagation on the closed contour of the Sagnac effect. Concluding remarks are given in Section VI.

\section{Describing Light Propagation On A Moving Closed Contour}

According to many physicists (e.g., [13], [14]), Einstein synchronization procedure introduces an indeterminateness to the one-way speed of light, which is related to the conventionality of clock synchronization. Then, within the scenario of relativistic theories where time depends on an arbitrary synchronization parameter $\varepsilon$ [13], we may use the following coordinate transformations between two inertial frames of reference, $S^{\prime}$ and $S$, in relative motion with velocity $v=i v$

$$
t^{\prime}=\frac{t}{\gamma}-\frac{\varepsilon x^{\prime}}{c^{2}} ; \quad x^{\prime}=\gamma(x-v t) ; y^{\prime}=y ; z^{-}=z
$$

Depending on the values of $\varepsilon$ in (1), we are met with infinite possible transformations but, fixing the value of $\varepsilon$, for simplicity we consider here the two most common transformations:

$$
L T(\varepsilon=v) \quad t^{\prime}=\gamma\left(t-\frac{v x}{c^{2}}\right) ; x^{\prime}=\gamma(x-v t) ; c^{-}=c
$$

$\operatorname{LTA}(\varepsilon=0) \quad t^{\prime}=\frac{t}{\gamma} ; x^{\prime}=\gamma(x-v t) ; c^{-}=\gamma^{2}(c-v)$.

In (2) the transformations $y^{\prime}=y ; z^{\prime}=z$ is understood. The factor $\gamma=\left(1-v^{2} / c^{2}\right)^{-1 / 2}$ depends on $v$ and LT stands for the Lorentz transformations based on standard synchrony and 
relative simultaneity $(\varepsilon=v)$. LTA stands for the Lorentz transformations based on absolute synchrony and simultaneity $(\varepsilon=0)$. On the right-hand-side of $(2)$, the speed $c^{\prime}=d x^{\prime} / d t^{\prime}$ is given for the special case of light speed $c$ in the direction of $v$ in frame $S$.

The LTA (or ALT, in [9]) are known in the literature also as the Tangherlini-Selleri transformations [15], [3], [16] and are formally the same as the ones used by various authors [6], [2], [9].

To point out the arbitrariness of the light speed, we write its explicit dependence on $\varepsilon$,

$$
c^{\prime}=c^{\prime}(\varepsilon)=\frac{d x^{\prime}}{d t^{\prime}}=\frac{c}{1+v / c-\varepsilon / c}
$$

easily derived from (1) assuming $c=d x / d t$ in $S$.

\section{AN EXAMPLE OF Light PROPAGATION ON A MOVING Closed CONTOUR}

In this Section we consider an ideal moving closed contour that is different from that of the Sagnac effect. However, as in the case of the Sagnac effect, our contour is closed in the sense that light (a photon) traveling on the contour starts from the measuring device (clock $\mathrm{C}^{*}$ ) and returns to it in the observable proper time interval $\tau_{\text {round }}$. Our basic assumption is that light propagates in the isotropic empty space of frame $S$, which can be thought of coinciding with the preferred frame of the LTA. Then, in $S$ the one-way speed of light $c$ can be used to synchronize distant clocks and measure the relative velocity $v$.

Our thought experiment involves a photon sent along a rod OB, stationary in $S$ (Fig. 1 a), from the position of clock $\mathrm{C}^{*} \equiv \mathrm{O}$ at the rod front end $\mathrm{O}$ to the rod back end $\mathrm{B} \equiv \mathrm{B}^{\prime}$ (Fig. $1 \mathrm{~b})$ and, after reflection from a mirror at $\mathrm{B} \equiv \mathrm{B}^{\prime}$, returning along the $\operatorname{rod} \mathrm{O}^{\prime} \mathrm{B}^{\prime}=L_{0}$ comoving with $S^{\prime}$ (Fig. 1 c). The front-end $\mathrm{O}^{\prime}$ of rod $\mathrm{O}^{\prime} \mathrm{B}^{\prime}$ passes the position of clock $\mathrm{C}^{*}$ at the same instant $(t=0)$ at which the photon reaches the back end $\mathrm{B}^{\prime}$ (Fig. $1 \mathrm{~b}$ ), and clock $\mathrm{C}^{*}$ is accelerated to comove now with the rod end $\mathrm{O}^{\prime}$. Finally, in its way back to $\mathrm{C}^{*} \equiv \mathrm{O}^{\prime}$ (Fig. $1 \mathrm{c}$ ), photon and clock meet at $\mathrm{O}^{\prime}$ after the time interval $\tau_{\text {round }}$.

As shown in Fig. $1 \mathrm{~b}$, the rod in motion $\mathrm{O}^{\prime} \mathrm{B}^{\prime}$ has the contracted length $L_{0} / \gamma$ relative to frame $S$ and the front and back ends of rod $\mathrm{OB}$ and $\mathrm{O}^{\prime} \mathrm{B}^{\prime}$ are made to coincide at $t=0$ with $\mathrm{OB}=L_{0} / \gamma$. Thus, at speed $c$ in $S$, the photon reaches point $\mathrm{B} \equiv \mathrm{B}^{\prime}$ at the distance $x_{B}=L_{o} / \gamma$ in the time interval,

$$
\tau_{\text {out }}=\frac{L_{0}}{\gamma c}
$$

When the origins $\mathrm{O}$ and $\mathrm{O}^{\prime}$ coincide (Fig. $1 \mathrm{~b}$ ) the clocks at the origins are set at $t=t^{\prime}=0$. At $t=0$ and simultaneously in $S$, clock $\mathrm{C}^{*}$ faces $\mathrm{O}^{-}$while the photon is at $\mathrm{B} \equiv \mathrm{B}^{\prime}$.

With clock $\mathrm{C}^{*}$ fixed to rod $\mathrm{O}^{\prime} \mathrm{B}^{\prime}$ in the photon return-trip, relative to $\mathrm{C}^{*}$ the total "ground" path length of the closed contour covered by the photon along the two rods in the time $\tau_{\text {round }}$ is,

$L_{t o t}=C^{*} B+B^{\prime} C^{*}=O B+B^{\prime} O^{\prime}=\frac{L_{0}}{\gamma}+L_{0} \simeq 2 L_{0}$ as apparent from Fig. $1 \mathrm{~b}$.

From (1) we find after some algebra that the position of the photon in frame $S^{\prime}$ at $t^{\prime}=0$ depends on $\varepsilon$ and is,

$$
x_{p h}^{\prime}\left(t^{\prime}=0 ; \varepsilon\right)=x_{0 p h}^{\prime}(\varepsilon)=L^{\prime}=\frac{L_{0}}{1+\left(\varepsilon / c^{2}\right) \gamma^{2}(c+v)}
$$

In changing its state of motion while passing from $S$ at $\mathrm{O}$ to $S^{\prime}$ at $\mathrm{O}^{\prime}$, clock $\mathrm{C}^{*}$ must have taken a finite time interval $\eta$. Moreover, there is also a time delay of the clock due to the effect of its acceleration while passing from frame $S$ to $S^{\prime}$, which can be taken into account and is of second order in $v / c$. When, after the interval $\eta, \mathrm{C}^{*}$ is finally comoving with $S^{\prime}$, the photon has moved toward $\mathrm{C}^{*} \equiv \mathrm{O}^{\prime}$ by $c^{\prime} \eta$ and, thus, is at the position $x_{p h}^{\prime}\left(t^{\prime}=\eta ; \varepsilon\right)=x_{0 p h}^{\prime}(\varepsilon)-c^{\prime} \eta$. Yet, for our example we may assume that $\mathrm{OB} \simeq \mathrm{O}^{\prime} \mathrm{B}^{\prime} \simeq L_{0}$ is large enough to have $c^{\prime} \eta \simeq c \eta<<c \tau_{\text {round }} \simeq 2 L_{0}$ and it is reasonable to neglect $\eta<<\tau_{\text {round }}$ and the time delay due to acceleration. Then, neglecting the small distance $c^{\prime} \eta$, we see that the position $x_{0 p h}^{\prime}(\varepsilon)$ of the photon at $t^{\prime} \simeq \eta \simeq 0$ does not depend on the acceleration of clock $\mathrm{C}^{*}$ but is determined by the choice of the synchronization adopted, regardless that $\mathrm{C}^{*}$ accelerates or not. Thus, the following calculations can be performed keeping in flat spacetime - (analogous approximations have been made in the discussion of the "linear" Sagnac effect in [2]. In fact, as mentioned by Malykin [7] in the context of the interpretation of the Sagnac effect with the General Theory of Relativity, "the Sagnac effect relates to the category of kinematic effects unrelated to any force").

a)

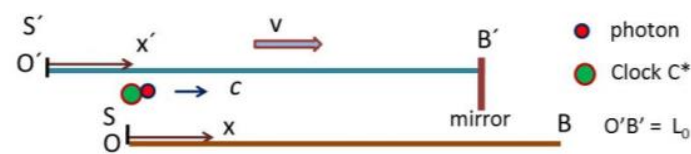

b)

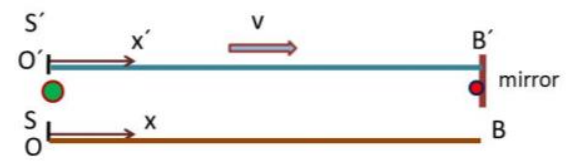

c)

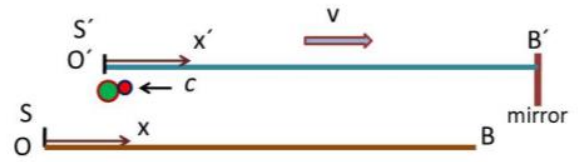

Fig. 1. Rod $\mathrm{O}^{-} \mathrm{B}^{-}$, stationary in frame $S^{-}$, is moving with velocity $v$ relative to frame $S$.

a) As seen from frame $S$, the photon starts its out trip from $\mathrm{O} \equiv \mathrm{C}^{*}$ and, after leaving clock $\mathrm{C}^{*}$, travels at speed $c$ toward $\mathrm{B}$ in the time $\tau_{\text {out }}$.

b) When the photon reaches $\mathrm{B} \equiv \mathrm{B}^{\prime}$, simultaneously in $S$ clock $\mathrm{C}^{*}$ faces $\mathrm{O}^{\prime}$. At this instant, when the origins $\mathrm{O}$ and $\mathrm{O}^{\prime}$ coincide at $t=t^{\prime}=0$, clock $\mathrm{C}^{*}$ starts comoving with $\mathrm{O}^{\prime}$, while the photon is being reflected at $\mathrm{B} \equiv \mathrm{B}^{\prime}$. c) From $\mathrm{B}^{\prime}$, the photon gets back to clock $\mathrm{C}^{*} \equiv \mathrm{O}^{\prime}$ in the return-trip time $t_{\text {ret }}$.

\section{A. Calculating the Round-trip Time $\tau_{\text {round }}$ from the Single Inertial Frame $S$}

In frame $S$ the photon speed is $c$ and, after reflection at $\mathrm{B}^{\prime}$ (Fig. $1 \mathrm{~b}$ ), at time $t$, its position is $L_{0} / \gamma-c t$ and the position of $\mathrm{C}^{*} \equiv \mathrm{O}^{\prime}$ is $v t$. Hence, photon and clock meet again when $L_{0} / \gamma-c t=v t$, after the time interval,

$$
t_{r e t}=\frac{L_{0}}{\gamma(c+v)}
$$


With the proper time $\tau$ of clock $\mathrm{C}^{*} \equiv \mathrm{O}^{\prime}$ related to $t$ by $\tau=t^{\prime}=t / \gamma \geq 0$, by means of (7) we may write,

$$
\tau_{\text {ret }}=t^{\prime}{ }_{r e t}=\frac{t_{r e t}}{\gamma}=\frac{L_{0}}{\gamma^{2}(c+v)}=\frac{L_{0}(1-v / c)}{c}
$$

Then, the measured round-trip proper time is,

$$
\begin{gathered}
\tau_{\text {round }}=\tau_{\text {out }}+\tau_{\text {ret }} \\
=\frac{L_{0}}{\gamma c}+\frac{L_{0}}{\gamma^{2}(c+v)}=\frac{L_{0}}{\gamma c}+\frac{L_{0}(1-v / c)}{c} \simeq \frac{2 L_{0}}{(c+v / 2)}
\end{gathered}
$$

where $\tau_{\text {out }}$ and $\tau_{\text {ret }}$ are observables measured separately in frame $S$ and $S^{\prime}$, respectively.

In (9) the closed contour $L_{\text {tot }} \simeq 2 L_{0}$ of (5) is covered by the photon at the average speed $c+v / 2>c$.

Relative to the single frame $S$, the total distance covered at speed $c$ is $\simeq L_{O}+L_{O}(1-v / c)=2 L_{O}(1-v / 2 c)$. In $S$ the return distance $\simeq L_{o}(1-v / c)$ is shorter than the outward path $\simeq L_{0}$ because $\mathrm{C}^{*} \equiv \mathrm{O}^{\prime}$ is moving toward the approaching photon. However, relative to $\mathrm{C}^{*} \equiv \mathrm{O}^{\prime}$ on $S^{\prime}$ the return "ground" path is $\simeq L_{0}$ and is covered in nearly the same interval $t_{\text {ret }}^{\prime} \simeq t_{\text {ret }}$. Thus, as derived below, the one-way speed in $S^{-}$is expected to be $c^{\prime} \simeq L_{0} / t^{\prime}$ ret $\simeq c+v$, i.e., greater than $c$.

The observable result (9) can be derived without problems from the single frame $S, S^{\prime}$, or any other inertial frame where the speed of light is assumed to be $c$. However, for a closed contour where a section is moving, the assumption that the light speed is $c$ in the chosen single frame, does not reveal that the local light speed is still $c$ (invariant) on the inertial frame comoving with the section in motion. Since our aim is to check that, relative to clock $C^{*}$, every section of the closed contour is being covered by the photon at the local speed consistent with the synchrony adopted, the local speed and corresponding path covered need to be evaluated separately on frames $S$ and $S^{\prime}$, with the approach adopted above.

In any case, it can be shown that, as long as the one-way speed is $c$ in frame $S$, our considerations are valid even when clock $C^{*}$ remains on frame $S$ during the time $\tau_{\text {round }}$ and $\tau_{\text {ret }}$ is measured by the clock of $S^{\prime}$ comoving with $\mathrm{O}^{\prime}$ (see the equivalent example of the linear Sagnac effect discussed in [2]).

\section{The Role of SimUltaneity IN DESCRIBING FROM $S^{\prime}$ Light PROPAGATION IN THE RETURN TRIP FROM B' TO $\mathrm{C}^{*}$}

If, after being reflected at $\mathrm{B}^{\prime}$, at $t^{\prime}=0$ the photon is at some point $x_{0 p h}^{\prime}=L^{\prime}$ along the rod and moving at local speed $c^{-}$ toward the origin $\mathrm{O}^{-}$, the kinematical constraint,

$$
\tau_{\text {ret }}=t_{L^{-}}^{\prime}=\frac{L^{\prime}}{c^{\prime}} \geq 0
$$

relates the time of flight $\tau_{\text {ret }}=t_{L^{\prime}}^{\prime}$, measured by clock $\mathrm{C}^{*} \equiv \mathrm{O}^{\prime}$ (starting from $t^{\prime}=0$ ), to the local speed $c^{\prime}$ and the path $L^{\prime}$ covered by the particle before reaching $C^{*}$. Since the measured $\tau_{\text {ret }}=t_{L^{\prime}}^{\prime}$ is known (8), the ratio $L^{\prime} / c^{\prime}$ is determined by (10).

\section{A. Determining $\varepsilon$ by Requiring Light to Cover the Whole Ground Path of the Closed Contour in the interval $\tau_{\text {round }}$}

$\left(L^{\prime}=L_{0}\right)$. Expression (5) requires that the ground path length, covered by the photon traveling from $\mathrm{B}^{\prime}$ to $\mathrm{C}^{*} \equiv \mathrm{O}^{\prime}$ in the return trip, is $L_{t o t}-L_{0} / \gamma=B^{\prime} C^{*}=L^{\prime}=L_{0}$. Therefore, for light propagation on the closed contour, by setting $L^{\prime}=L_{0}$ in (6) we obtain,

$$
1+\left(\varepsilon / c^{2}\right) \gamma^{2}(c+v)=1 \quad \Rightarrow \quad \varepsilon=0 .
$$

For the photon moving in the $-x^{\prime}$ direction (velocity $c_{x}=-c$ in frame $S$ ), (3) and (11) give,

$$
c^{\prime}=\frac{c}{1-v / c}=\gamma^{2}(c+v) \text {. }
$$

Result (12) is consistent with the velocity composition of absolute simultaneity in (2).

The path effectively covered by the photon in frame $S^{\prime}$ is:

$$
B^{\prime} C^{*}=L^{\prime}=c^{\prime} \tau_{r e t}=\gamma^{2}(c+v) \tau_{r e t}=L_{0} .
$$

With conservation of simultaneity $(\varepsilon=0)$, the positions of photon and clock $\mathrm{C}^{*}$ in $S^{\prime}$ are shown in Fig. 2 a.

The motion of the photon can be seen in the diagram of Fig. 3 also, where the dotted lines refer qualitatively to light propagation in the case of $\varepsilon=0$ (axes $c t$ and $x$ not shown) of transformations LTA (2) preserving simultaneity.

The photon travels initially from $\mathrm{C}^{*}$ to $\mathrm{B} \equiv \mathrm{B}^{\prime}$ (diagram section $\left.\mathrm{P}_{\mathrm{i}}-\mathrm{B}^{\prime}\right)$ at speed $c$ for $S$ and $c^{\prime}=\gamma^{2}(c-v)$ for $S^{\prime}$.

The corresponding events $\left(\mathrm{E}:\left[(c t, x) ;\left(c t^{\prime}, x^{\prime}\right)\right]\right)$ are:

a) absolute simultaneity

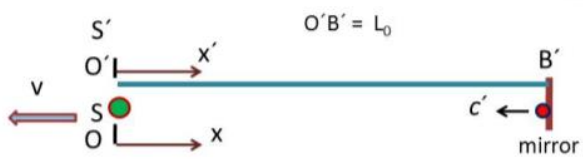

b) relative simultaneity

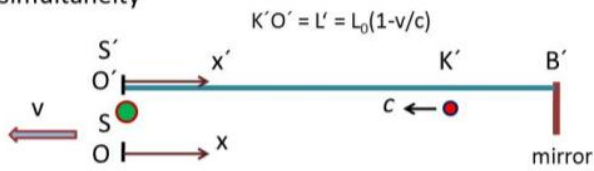

Fig. 2. Relative to $\operatorname{rod} \mathrm{O}^{\prime} \mathrm{B}^{\prime}$, stationary in $S^{\prime}$, frame $S$ is moving with velocity $\simeq-v$.

a) Absolute simultaneity $(\varepsilon=0)$. As seen from $S^{\prime}$, at $t^{\prime}=t=0$ clock $\mathrm{C}^{*}$ is now stationary at point $\mathrm{O}^{\prime}$ and, simultaneously, the photon is at $\mathrm{B}^{\prime}$.

Traveling at local speed $c^{\prime}=\gamma^{2}(c+v)$ the photon reaches clock $\mathrm{C}^{*}$ at $\mathrm{O}^{\prime}$ after the return-trip time $\tau_{\text {ret }}=\mathrm{B}^{\prime} \mathrm{O}^{\prime} / c^{\prime}=L_{0} / \mathrm{c}^{\prime}$.

b) Relative simultaneity $(\varepsilon=v / c)$. As seen from $S^{\prime}$, at $t^{\prime}=0$ the photon is at point $\mathrm{K}^{\prime}$ and, traveling at speed $c$ reaches clock $\mathrm{C}^{*}$ at $\mathrm{O}^{\prime}$ after the return-trip time $\tau_{r e t}=\mathrm{K}^{\prime} 0^{\prime} / c=L_{0}(1-\mathrm{v} / \mathrm{c}) / c$.

$\mathrm{P}_{\mathrm{i}}^{\prime} \quad\left[\left(-L_{0} / \gamma, 0\right) ;\left(-L_{0} / \gamma^{2}, v L_{0} / c\right)\right)$ and $\mathrm{P}_{\mathrm{B}}^{\prime}{ }^{\prime}\left[\left(0, L_{0} / \gamma\right) ;\left(0, L_{0}\right)\right)$ (not shown in the diagram). Thus, the photon is at $\mathrm{B} \equiv \mathrm{B}^{\prime}$ when $\mathrm{C}^{*}, \mathrm{O}^{\prime}$ and $\mathrm{O}$ coincide at $t^{\prime}=t=0$. From $\mathrm{B} \equiv \mathrm{B}^{\prime}$ the photon covers the ground path $\mathrm{B}^{\prime} \mathrm{C}^{*}=\mathrm{B}^{\prime} \mathrm{O}^{\prime}=L_{0}$ (diagram section $\left.\mathrm{B}^{\prime}-\mathrm{P}_{\mathrm{f}}\right)$ to reach $\mathrm{C}^{*}$ at $x^{\prime}=0$ in the measured interval $\tau_{\text {ret }}$ at speed $c^{\prime}=\gamma^{2}(c+v)$.

\section{B. The Value of $L^{\prime}$ Foreseen by the LT with Relative Simultaneity}

$\left(L^{\prime}=L_{0}(1-v / c)\right)$. The LT establish the photon initial 
position to be $x^{\prime}{ }_{0 p h}(\varepsilon=v)=L^{\prime}=L_{0}(1-v / c)$ in $S^{\prime}$. In fact, at $x_{\mathrm{B}}=L_{0} / \gamma$ and $t=0$ in $S$, the LT (2) foresee the time discontinuity $-\delta t^{\prime}=-\gamma v x_{\mathrm{B}} / c^{2}=-L_{0} v / c^{2}$ between clocks of $S^{\prime}$ and $S$. Thus, as seen from $S^{\prime}$, the photon has reached point $\mathrm{B}$ in the past $\left(t^{\prime}=-\delta t^{\prime}\right)$. Since, at speed $c$, the photon has covered the path $c \delta t^{\prime}=L_{0}-L^{\prime}$ in the interval $\delta t^{\prime}$, at the present time $t^{\prime}=0$, the photon is already at $\mathrm{K}^{\prime}$ at the distance $L^{\prime}<L_{0}$ from $\mathrm{C}^{*}$, as shown in Fig. 2 b.

Then, with $\varepsilon=v$ in (6) and $c^{\prime}=c$, the path effectively covered in frame $S^{\prime}$ at speed $c$ is:

$$
K^{\prime} C^{*}=L^{\prime}=c \tau_{r e t}=L_{0}\left(1-\frac{v}{c}\right)=L_{0}-c \delta t^{\prime}<L_{0}
$$

The difference $L_{0}-L^{\prime}=L_{0} v / c=c \delta t^{\prime}$ is due to nonconservation of simultaneity between frame $S$ and $S^{\prime}$.

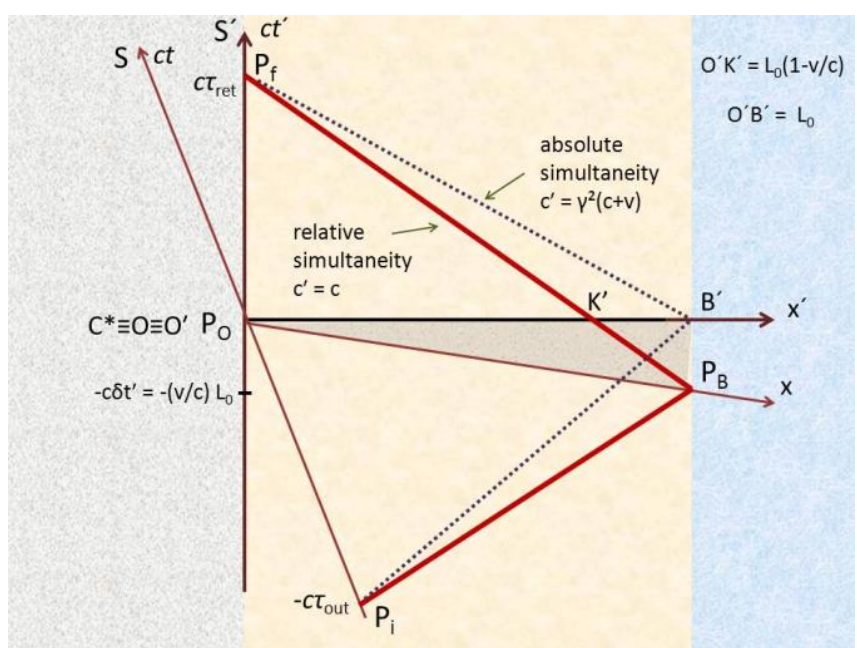

Fig. 3. Spacetime diagram of light propagation Absolute simultaneity $(\varepsilon=0)$ : Dotted lines (the $c t$ and $x$ axes

are not shown). As seen from $S^{\prime}$, the photon travels initially at speed $c^{\prime}$ $=\gamma^{2}(c-v)$ from $\mathrm{C}^{*}$ to $\mathrm{B}^{\prime}$ (diagram section $\left.\mathrm{P}_{\mathrm{i}}-\mathrm{B}^{\prime}\right)$. Then, from $\mathrm{B}^{\prime}$ at $t^{\prime}=0$, the photon covers the return path $\mathrm{B}^{\prime} \mathrm{C}^{*}=\mathrm{B}^{\prime} \mathrm{O}^{\prime}=L_{0}$ (diagram section $\mathrm{B}^{\prime}-\mathrm{P}_{\mathrm{f}}$ ) in the measured interval $\tau_{\text {ret }}$ at speed $c^{\prime}=\gamma^{2}(c+v)$.

Relative simultaneity $(\varepsilon=v / c)$ : The continuous line represents

the world line of a photon moving at speed $c$ from $\mathrm{P}_{\mathrm{i}}$ to $\mathrm{P}_{\mathrm{B}}, \mathrm{K}^{\prime}$, and ending at $\mathrm{P}_{\mathrm{f}}$ at $t^{\prime}=\tau_{\text {ret }}$. The events $\mathrm{P}_{\mathrm{O}}$ and $\mathrm{P}_{\mathrm{B}}$ are simultaneous in frame $S$, but not simultaneous in $S^{\prime}$.

In frame $S^{\prime}$ the events on segment $\mathrm{P}_{\mathrm{B}}-\mathrm{K}^{\prime}$ take place in the past $\left(t^{\prime}<0\right)$. Since the photon reaches $\mathrm{K}^{\prime}$ at $t^{\prime}=0$, the time interval $\delta t^{\prime}$ is not measured by clock $\mathrm{C}^{*}$ while comoving with $S^{\prime}$. Therefore, the measured $\tau_{\text {ret }}\left(t^{\prime} \geq 0\right)$ refers to the photon motion from $\mathrm{K}^{\prime}$ to $\mathrm{P}_{\mathrm{f}}$ only.

\section{Consequences of Adopting Standard Synchrony in $S^{-}$}

The Minkowski spacetime diagram of Fig. $3(\varepsilon \neq 0$, e.g., standard synchrony with $\varepsilon=v$ ) shows the photon world line in its round-trip.

Using the LT (2), the events $\left(\mathrm{E}:\left[(c t, x) ;\left(c t^{\prime}, x^{\prime}\right)\right]\right)$ are:

$\mathrm{P}_{\mathrm{i}}\left[\left(-L_{0} / \gamma, 0\right) ; \quad\left(-L_{0}, v L_{0} / c\right)\right], \quad \mathrm{P}_{\mathrm{O}}[(0,0) ;(0,0)], \quad \mathrm{P}_{\mathrm{B}}\left[\left(0, L_{0} / \gamma\right) ;(-\right.$ $\left.\left.v L_{0} / c, L_{0}\right)\right], \mathrm{P}_{\mathrm{f}}\left[\gamma L_{0}(1-v / c)(1, v / c) ;\left(L_{0}(1-v / c), 0\right)\right]$.

In the out trip the ground path $\mathrm{C}^{*} \mathrm{~B}=\mathrm{OB}$ (diagram section $\left.\mathrm{P}_{\mathrm{i}}-\mathrm{P}_{\mathrm{B}}\right)$ is traversed by the photon at speed $c$ in the time interval $\tau_{\text {out }}$ measured on frame $\mathrm{S}$.

The remaining return ground path $\mathrm{BO}^{\prime}=\mathrm{BC}^{*}$ (diagram section $\mathrm{P}_{\mathrm{B}}-\mathrm{P}_{\mathrm{f}}=\mathrm{P}_{\mathrm{B}}-\mathrm{K}^{\prime}+\mathrm{K}^{\prime}-\mathrm{P}_{\mathrm{f}}$ ) is covered by the photon in the time interval $\tau_{r e t}+\delta t^{\prime}$, being $\tau_{\text {ret }}$ measured by $\mathrm{C}^{*}$ on frame $S^{\prime}$ for $\mathrm{t}^{\prime} \geq 0$. The region of triangle defined by $\mathrm{P}_{\mathrm{O}}, \mathrm{P}_{\mathrm{B}}, \mathrm{B}^{\prime}$ limits the gap in the world line (diagram section $\mathrm{P}_{\mathrm{B}}-\mathrm{K}^{\prime}$ ) related to the time discontinuity $\delta t^{\prime}$ due to nonconservation of simultaneity.

Thus, if we choose to adopt the LT, there is a price to pay for it. In fact, with $\varepsilon=v$ and $L^{\prime}=L_{0}(1-v / c)=\mathrm{K}^{\prime} \mathrm{C}^{*}$ (Fig. 2 b), the round-trip time measured by clock $C^{*}$ may be expressed as,

$$
\begin{aligned}
\tau_{\text {round }} & =\tau_{\text {out }}+\tau_{\text {ret }}=\frac{C^{*} B}{c}+\frac{K^{\prime} C^{*}}{c} \\
& =\frac{L_{0} / \gamma}{c}+\frac{L^{\prime}}{c}=\frac{L_{0} / \gamma}{c}+\frac{L_{0}(1-v / c)}{c} .
\end{aligned}
$$

Interpreting $\tau_{\text {round }}$ with relative simultaneity, the correct result (15) is obtained only if the time interval $\delta t^{\prime}$, taken by the photon to cover section $\mathrm{BK}^{\prime}$ (diagram section $\mathrm{P}_{\mathrm{B}}-\mathrm{K}^{\prime}$ ), is not included in (15). In fact, the photon covers $\mathrm{BK}^{\prime}$ (diagram section $\left.\mathrm{P}_{\mathrm{B}}-\mathrm{K}^{\prime}\right)$ in the past $\left(t^{\prime}<0\right)$ and, in the interval $\tau_{\text {ret }}\left(t^{\prime} \geq\right.$ 0 ), the photon covers at speed $c$ section $\mathrm{K}^{\prime} \mathrm{C}^{*}$ (diagram section $\mathrm{K}^{\prime}-\mathrm{P}_{\mathrm{f}}$ ) only.

By requiring that be covered at speed $c$, the return path $\mathrm{B}^{\prime} \mathrm{C}^{*}$ must be reduced to $\mathrm{K}^{\prime} \mathrm{C}^{*}=L_{0}(1-v / c)<L_{0}$, because it is physically impossible to cover at speed $c$ the whole section $\mathrm{B}^{\prime} \mathrm{C}^{*}=L_{0}$ in the interval $\tau_{\text {ret }}$. Thus, if light propagates at local speed $c$, the contour effectively covered in the interval $\tau_{\text {round }}$ is open and given by $L_{0} / \gamma+L_{0}(1-v / c) \simeq 2 L_{0}-c \delta t^{\prime}$ $<2 L_{0}$. If path $\mathrm{B}^{\prime} \mathrm{C}^{*}$ is not shortened, we have to add to (15) the time interval $\delta t^{\prime}=\mathrm{B}^{\prime} \mathrm{K}^{\prime} / c=L_{0} v / c^{2}$ (time gap). Then, (15) becomes $\tau_{\text {round }}+\delta t^{-} \simeq 2 L_{0} / c$, consistent with a local speed $c$ along the whole closed contour, but in disagreement with observation.

It follows that, for a consistent description of light propagation along the whole closed contour (requiring $L^{\prime}=$ $L_{0}$ ), if the local speed of light is $c$ on frame $S$, on the relatively moving section $\mathrm{B}^{\prime} \mathrm{C}^{*}$ the speed $c^{\prime}$ is not arbitrary but determined to be $c^{\prime-}=\gamma^{2}(c+v)$.

\section{SOME REMARKS ON THE SAGNAC EFFECT}

In relation to the interpretation of the circular Sagnac effect [11] we cite the works of Selleri [3], who makes use of transformations LTA (2). Selleri [3] claims that, by applying the LT to the circular platform (rotating at angular speed $\omega$ ) where light propagates, we are met with inconsistencies, independently of the radius $r$ of the platform. To prove his claim, Selleri considers the special case when the radius $r$ of the platform tends to infinity, keeping constant the tangential speed $v=\omega r$. Then, Selleri shows that a consistent interpretation of the Sagnac effect in this special case is obtained by adopting the LTA based on conservation of simultaneity, with the speed of light on the rotating platform given by the average speed $(\simeq c \pm v$, depending on the direction of motion).

The inconsistency revealed by Selleri can be related to the discontinuity in time mentioned by Landau and Lifshitz [10], which has been pointed out by Klauber [1] and Gift [6] for the case of Einstein synchronization applied on a rotating disc, showing that it amounts to a clock being out of synchronization with itself. Furthermore, extensive considerations supporting conservation of simultaneity have been made by Lundberg [4] and Field [5]. Moreover, and always in the context of the Sagnac effect, Kipreos and Balachandran [9] show that diverse relativistic optical and 
nonoptical rotational data support the use of the absolute Lorentz transformations, rather than the standard LT.

A different approach to the Sagnac effect is given by Hajra [8] taking into account the Coriolis effect.

Another example of a moving closed contour is given by the equivalent linearized (or "linear") version of the circular Sagnac effect, proved experimentally by Wang et al. [17] in 2003. As shown in [2], for the linear effect apply the same conclusions of Sections III and IV.

Let us now go back to the example of Fig. 1 and try to link the results obtained with those of the circular Sagnac effect. In our example we considered the case when clock $\mathrm{C}^{*}$, initially at rest in frame $S$, where the one-way speed of light is $c$, at $t=t^{\prime}=0$ changes its velocity relative to $S$ and starts moving at speed $v$ toward the approaching photon. The result is that, if the contour covered is closed, in the return trip the photon speed relative to $C^{*}$ is $c^{\prime}=\gamma^{2}(c+v)$, as derived by Selleri for the Sagnac effect. Let us consider the analogous case of a photon on the rotating platform, where the corotating clock $\mathrm{C}^{*}$ has the peripheral speed $v$ relative to the laboratory frame $S$ where the center of the platform is stationary. If the photon is counter-propagating along the rim of the platform of rest length $\gamma 2 \pi r$, the clock is approaching the photon and, thus, the photon speed relative to $\mathrm{C}^{*}$ is $c^{\prime}$ greater than $c$. In fact, in this case the observable round-trip time is,

$$
\tau_{\text {round }}=\frac{2 \pi r}{\gamma(c+v)}=\frac{2 \gamma \pi r}{\gamma^{2}(c+v)}=\frac{2 \gamma \pi r(1-v / c)}{c}
$$

and, in analogy with the results of Sections III and IV, expression (16) implies that,

$$
2 \gamma \pi r=c^{-} \tau_{\text {round }}=\gamma^{2}(c+v) \tau_{\text {round }},
$$

indicating that the closed circular path is covered by the photon at the speed $c^{\prime}=\gamma^{2}(c+v)$, consistent with the velocity composition of the LTA based on conservation of simultaneity.

Of course, we may adopt standard synchrony and require that the local speed be $c$ along the closed contour. Then, in analogy with result of Section IV, from the last term of (16) the path effectively covered at speed $c$ in the interval $\tau_{\text {round }}$,

$$
2 \gamma \pi r\left(1-\frac{v}{c}\right)=c \tau_{\text {round }}=2 \gamma \pi r-c \delta t^{\prime}<2 \gamma \pi r,
$$

is necessarily open because the section $c \delta t^{\prime}=(v / c) 2 \gamma \pi r$ is not covered by the photon in the interval $\tau_{\text {round }}$.

Thus, given that result (18) reflects the unfeasibility of performing Einstein synchronization on a closed contour [1][6], [10], a consistent description of light propagation in the circular Sagnac effect requires conservation of simultaneity with the local speed given by $c^{\prime}=\gamma^{2}(c+v)$, as in (17).

\section{CONCLUSIONS}

At the time when in 1913 Sagnac [11] performed his experiment, physicists were using the Galilean transformations. Assuming that the one-way speed of light is $c$ in the laboratory frame of the experiment, an observer comoving with clock $\mathrm{C}^{*}$ at peripheral speed $v=\omega r$ on the rotating platform was expected to see the speeds $c^{\prime}=c+v$ or $c^{\prime}=c-v$ for light propagating along the rim of the platform. Since his experiment confirmed the Galilean composition of velocities, Sagnac concluded that it ruled out the invariance of the speed of light, $c^{\prime}=c$, postulated by Einstein. Although the LT and the invariance of $c$ were later widely accepted, in 1996-7 the works of Selleri [3] showed that a consistent interpretation of the circular Sagnac effect requires coordinates transformations that conserve simultaneity (e.g., LTA) and, thus, Selleri claimed that the LT based on relative simultaneity had to be abandoned. Nevertheless, the previous work of Mansouri and Sexl [13] of 1977 suggested that the one-way speed of light is arbitrary and that the LT and LTA are physically equivalent because they differ by an arbitrary synchronization constant. The approach of Mansouri and Sexl has been used by several physicists to resolve the paradoxes of special relativity that arise when using the LT. The solution involves solving the paradoxes using the LTA (based on conservation of simultaneity) and then claiming wrongly that the paradoxes are solved with the LT (based on relative simultaneity) also, on the basis of the physical equivalence of the LTA with the LT due to the arbitrariness of synchronization.

However, in agreement with the analysis of Selleri [3], Gift [6], Lundberg [4], Field [5], Kipreos and Balachandran [9], and many other physicists (e.g., [1], [2], [16]), we have shown in the previous Sections that the interpretation of our example (and the Sagnac effect) requires conservation of simultaneity, discarding the LT. Therefore, although the LT and LTA might be equivalent for describing light propagation on open contours, our claim is that the LT and the LTA are not physically equivalent in the case of light propagation along moving closed contours where no synchronization is needed for the single clock measuring the round-trip time.

The use of the LTA has been considered by Kassner [18] and Lee [19]. However, both authors assume erroneously in their works that the LTA and the LT are physically equivalent. In fact, in its ways of describing the Sagnac effect, Kassner [18] states: "We conclude that Einstein synchronization fails when performed along a path around the full circle". Then, to dismiss Selleri's paradox and the difficulties in interpreting Sagnac's effect with the LT based on standard synchrony, Kassner introduces the unphysical concept of "time gap", criticized by Gift [6] and other authors [2]. Finally, Kassner interprets the Sagnac effect consistently by switching from the LT to the LTA (i.e., passing from relative to absolute synchrony) justifying the shift on the basis of the liberty in the choice of synchronization. Nevertheless, Kassner did not verify the path length covered by the light at the local speed $c$ and, furthermore, it is not clear how, in this case, the LT and the invariance of $c$ can be assumed to be valid when, as Kassner himself argues, Einstein synchronization fails when performed along the closed path of the Sagnac effect.

Similar consideration can be extended to the arguments of Lee [19], who discusses special relativity in cylindrical spacetime. Lee [19] considers the case where the universe is closed in some spatial dimension like the surface of a cylinder and shows that the "undesirable consequences" that emerge with standard synchrony disappear by adopting conservation of simultaneity and a speed of light coinciding with the 
average speed $(\simeq c \pm v)$. In this case, from a conceptual physical perspective it is unclear how the LT can be considered equivalent to the LTA (as Lee assumes) when paradoxes and undesirable consequences emerge with the LT. Instead, what becomes apparent from the works of Kassner and Lee is that, for avoiding paradoxes or undesirable consequences, a consistent interpretation of physical phenomena requires the use of transformations based on conservation of simultaneity, ruling out the Lorentz transformations.

In conclusion, we have shown in the previous Sections that, by assuming the one-way speed of light to be $c$ on the rest frame $S$ of our example, the partial proper time intervals $\tau_{\text {out }}$ and $\tau_{\text {ret }}$ are observables measurable by clock $\mathrm{C}^{*}$. Application of the LT between sections in relative motion yields the undesirable consequence that, if the local speed is $c$ on one section (frame $S$ ), on the moving section (frame $S^{\prime}$ ) light covers at speed $c$ an open part only of the section. Thus, light propagation on the moving closed contour is not described consistently by adopting the LT with relative simultaneity. Instead, a consistent description of light propagation in our example (and the Sagnac effect) is provided by adopting transformations based on conservation of simultaneity.

\section{ACKNOWLEDGMENTS}

We wish to thank the International Center for Theoretical Physics (ICTP), Trieste, Italy, for the award of its Braingain program support.

\section{REFERENCES}

[1] R. D. Klauber, "Comments regarding recent articles on relativistically rotating frames", Am. J. Phys. 67(2), 158-159,1999; "Anomalies in Relativistic Rotation", Journal of Scientific Exploration, 16, 603-620, 2002.

[2] G. Spavieri, G. T. Gillies, E. Gaarder Haug, and A. Sanchez, "Light propagation and local speed in the linear Sagnac effect," Journal of Modern Optics, Open Access, nov. 2019; "The Sagnac effect and the role of simultaneity in relativity theory", Journal of Modern Optics, Open Access, feb. 2021.

[3] F. Selleri, "Noninvariant one-way velocity of light", Found. Phys. 26, 641, 1996; "Noninvariant One-Way Speed of Light and Locally Equivalent Reference Frames," Found. Phys. Lett. 10, 73-83, 1997.

[4] R. Lundberg, "Critique of the Einstein clock variable", Phys. Essays 32, 237, 2019; "Travelling light", Journal of Modern Optics, 68:14, 717-741, 2021, DOI: 10.1080/09500340.2021.1945154

[5] J. H. Field, "The Sagnac Effect and Transformations of Relative Velocities Between Inertial Frames" Fund. J. Modern Phys., Vol. 10, Issue 1: 1-30, 2017.

[6] S. J. G. Gift, " On the Selleri Transformations: Analysis of Recent Attempts by Kassner to Resolve Selleri's Paradox," Applied Physics Research, Vol. 7, No. 2, 2015.

[7] G. B. Malykin, "The Sagnac effect: correct and incorrect explanations", Physics - Uspekhi, 43 (12) 1229-1252, 2000.

[8] S. Hajra, "Spinning Earth and its Coriolis effect on the circuital light beams: Verification of the special relativity theory", Pramana $-J$. Phys., Indian Academy of Sciences, 87:71, 2016. DOI 10.1007/s12043016-1288-5.

[9] Edward T. Kipreos and Riju S. Balachandran, "An approach to directly probe simultaneity", Modern Physics Letters A, Vol. 31, No. 26, 1650157, 2016; "Assessment of the relativistic rotational transformations", Modern Physics Letters A, Vol. 36, No. 16, 2150113 , 2021.

[10] L. D. Landau and E. M. L. Lifshitz, The Classical Theory of Fields, Vol. 2, 236, Pergamon Press, Second English edition, 1962.
[11] G. Sagnac, "L'éther lumineux démotré par l'effet du vent relatif d'éther dans un intertféromètre en rotation uniforme," C. R. Acad. Sci., 157, 708-710, 1913 .

[12] E. J. Post, "Sagnac Effect", Rev. Mod. Phys. 39(2), 475-493, 1967.

[13] R. Mansouri, and R. U. Sexl, "A test theory of special relativity," Gen. Rel. Grav., 8: 497, 515, 809, 1977.

[14] R. de Abreu, and V. Guerra, "On the Consistency between the Assumption of a Special System of Reference and Special Relativity," Found. Phys., 36, 1826-1845 (2006); "The principle of relativity and the indeterminacy of special relativity," Eur. J. Phys. 29, 33, 2008.

[15] F. R. Tangherlini, "Galilean-Like Transformation Allowed by General Covariance and Consistent with Special Relativity," Nuovo Cimento Suppl., 20, 1, 1961.

[16] G. Spavieri, M. Rodriguez, and A. Sanchez, "Thought experiment discriminating Special Relativity from Preferred Frame Theories," J. Phys. Commun. 2, 085009, aug. 2018. doi.org/10.1088/23996528/aad5fa

[17] R. Wang, Y. Zhengb, A. Yaob, and D. Langley, "Modified Sagnac experiment for measuring travel-time difference between counterpropagating light beams in a uniformly moving fiber," Phys. Lett. A, 312, 7-10, 2003. R. Wang, Y. Zheng, and A. Yao, "Generalized Sagnac Effect", Phys. Rev. Lett. 93(14):143901, 2004.

[18] K. Kassner, "Spatial geometry of the rotating disk and its non-rotating counterpart", Am. J. Phys. 80, 772, 2012; "Ways to resolve Selleri's paradox," Am. J. Phys., 80, 1061, 2012.

[19] Chunghyoung Lee, "Simultaneity in cylindrical spacetime," Am. J. Phys. 88, 131, 2020.

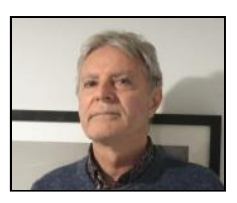

Gianfranco Spavieri was Born in Verona (Italy) on the $28^{\text {th }}$ of April, 1942. He received his education in Milan (Italy), obtaining the degree of Doctor of Nuclear Engineering from the Polytechnic of Milan in 1968, and, after changing field, the degree of Master of Science in Physics from the University of California at Santa Cruz

(US) in 1972

First as teaching assistant and later as assistant professor, he taught and carried out research in physics at universities and research institutions of California, Italy, Arizona, and Venezuela. Since 1975 he is a faculty member of the Physics Department of the University of the Andes, Mérida (Venezuela). Among his publications of over 150 specialized physics articles in international journals there are also 2 books dealing with science, art and myth.

Dr. Spavieri is currently Professor of Physics at the Center of Fundamental Physics (University of the Andes, Venezuela) of which is a co-founder. He has acted as director of several academic theses at the undergraduate and graduate levels, and as referee of many international physics journals. He carried out several research projects sponsored by his university and other research institutions and was granted various national and international awards. Research interests have been, and currently are, fundamental physics, quantum physics, classical electrodynamics, and relativity. 